BOUNDARY PLASMA MODELLING FOR ITER

\author{
Progress Report
}

for Period July 1, 1992 - December 31, 1992

B. J. Braams

Courant Institute of Mathematical Sciences

New York University

New York, NY 10012

January 1993

Prepared for

THE U. S. DEPARTMENT OF ENERGY

Cangen

FED $1>1993$

$0 \Omega T_{1}$

AGREEMENT NUMBER DOE-FG02-92ER54165

\title{
NOTICE
}

This report was prepared as an account of work sponsored by the United States Government. Neither the United States nor the Department of Energy, nor any of their employees, nor any of their contractors, subcontractors, or their employees, makes any warranty, express or implied, or assumes any legal liability or responsibility for the accuracy, completeness, or usefulness of any information, apparatus, product or process disclosed or represents that it 8 use would not infringe privately-owned rights. 
Abstract. We have developed computer programs to model the effect of nonaxisymmetric magnetic perturbations upon divertor heat load, and have explored what kind of externally applied perturbations are the most effective for heat load reduction without destroying core plasma confinement. We find that a carefully tuned set of coils located about $0.3 \mathrm{~m}$ outside the separatrix can be used to spread the heat load over about $0.1 \mathrm{~m}$ perpendicular to flux surfaces at the ITER divertor plate, even at a very low level of anomalous cross-field heat transport. As such a spreading would significantly extend the permissible regime of operation for ITER, we recommend that this study be pursued at the level of detail required for engineering design. In other work under this grant we are in the process of modifying the B2 code to handle correctly a non-orthogonal geometry.

Non-Axisymmetric Magnetic Perturbations. The heat load on a divertor target plate is spread over a wider region as the magnitude of the radial thermal conductivity in the edge plasma is increased. This observation gives rise to speculation that "ergodization" of the edge plasma, through deliberate external magnetic field perturbations, could be employed to reduce the peak heat load on the divertor target plate in ITER. As ergodization does not apply in the region of open field lines, this concept morits a sceptical view and careful numerical study. Until now there had been no detailed numerical simulation of transport in a three-dimensional magnetic geometry relevant to this concept.

The transport process in a tokamak plasma is, of course, highly anisotropic. For relevant edge plasma parameters $\left(T_{\mathrm{e}} \simeq 200 \mathrm{eV}\right.$ and $\left.n_{\mathrm{e}} \simeq 3 \times 10^{19} / \mathrm{m}^{3}\right)$ the ratio of parallel (classical) to perpendicular (anomalous) thermal diffusivity is about $10^{8}$. This poses no great difficulty for try sport simulations in one-dimensional or twodimensional geometry, when one coo dinate is aligned with flux surfaces, but in threedimensional geometry with a general magnetic field such a coordinate system does not exist, and transport simulations by finite-difference, finite-volume or finite-element techniques become very difficult due to the problem of false numerical diffusion.

We decided to employ a grid-free random-walk method to solve a highly anisotropic model heat transport equation in a realistic perturbed tokamak magnetic field. The walkers are launched in the core plasma and make small steps across the magnetic field and long steps along the field lines; the latter steps are evaluated by accurate field line integration. The partial differential equation solved is $\nabla \cdot \mathbf{Q}=0$ where $\mathbf{Q}=-\kappa_{\|} \nabla_{\|} T-\kappa_{0} \nabla T$. The distribution of points where the walkers cross the boundary is interpreted as the heat load on the boundary. This is a model equation, because we do not consider the dependence of $\kappa_{\|}$on temperature, nor do we solve for a consistent density profile. Only the (constant) ratio of parallel to perpendicular diffusivity is important.

Since thousands of walkers have to be followed to obtain accurate statistics, and since each walker can wander around for thousands of steps before reaching the boundary, it is essential to have a very efficient representation of the magnetic field. Our work evolved through two stages in this regard. At first we relied on a set of 
local toroidal harmonic expansions for the axisymmetric part of the magnetic field, and on a representation as a sum of dipole fields for the perturbation. Later, following a suggestion of J. Nührenberg of IPP Garching, we used local Hermite interpolation on data representing the function values and first derivatives of all components of the magnetic field on a regular mesh.

The present random-walk calculation shares some features with "deep penetration" problems in Monte Carlo neutron transport studies. Our walkers are launched in the interior of the plasma, but all that we are interested in is the distribution of points on the boundary at which, after many steps, they eventually leave the domain of the calculation. The efficiency of this random walk calculation is greatly improved by judicious use of splitting and Russian roulette. We divide the computational region up into zones, with zone boundaries defined by the equilibrium magnetic flux surfaces. On each zone a "target weight" is defined; the target weights decrease exponentially as we move towards the outside of the confinement region. The particles are launched in the inside with unit weight; at any stage, if a particle weight exceeds the target weight of the region it is in by some factor then the particle is split in two with each new particle carrying half the weight; on the other hand, if a particle weight is less than the target weight by some factor then it is terminated with probability 0.5 , doubling its weight if it survives.

We performed calculations for a magnetic equilibrium representing an up-down symmetric double-null SSAT/TPX (equilibrium 92047, July 1992, c 1 1rtesy of R. Bulmer of LLNL). This device has major radius $R_{0}=2.25 \mathrm{~m}$. All our calculations scale linearly with geometry, so we obtain results relevant to ITER by multiplying all geometry data by a factor of abou 3 . The equilibrium is shown in Fig. 1.

The analytical representation of the equilibrium magnetic field that is used for our calculations is obtained via a wire model for the plasma current. That representation is accurate near and outside the separatrix. It degrades in the core plasma, but that is immaterial for our studies. The magnetic geometry of the wire model is showi in Fig. 2.

In engineering practice we expect that the magnetic field perturbation would be created by a large set of coils mounted on or inside the vacuum vessel. As we are not well placed at NYU to perform detailed design, we chose to represent the perturbation as a superposition of magnetic dipole fields. The dipoles were located along several rings outside the plasma. The number and placement of these rings, the dominant toroidal mode number of the perturbation, the toroidal phase difference between the perturbation on different rings, and the amplitude of the perturbation were all subject to optimization. Here we present results only for the most favourable configuration studied, which involved six toroidal rings (in two pairs of three symmetric with respect to the equatorial plane), located as indicated on Fig. 2. The perturbation has dominant toroidal mode number equal to 8 . The maximum amplitude of the perturbed magnetic field at the separatrix is 3.2 percent of the local equilibrium toroidal magnetic field. 
Figure 3 shows a Poincaré plot of the magnetic field structure under the influence of this perturbation. The perturbation has destroyed the outermost $18 \%$ of the radius of good flux surfaces when measured on the inboard side of the torus. Due to the Shafranov shitt this translates to a destruction of the outermost $14 \%$ of the radius of good flux surfaces on average. Somewhat arbitrarily we view this as a maximum permissible level of perturbation. In all our optimization studies we selected the amplitude of the perturbing field to result in this same level of destruction of good flux surfaces.

In Fig. 4 is shown the distribution of points on the lower boundary where the random walkers leave the domain. Without perturbation the corresponding picture is composed of just one ring, widened only by the process of microscopic physical diffusion. Under the influence of the perturbation the simple ring is replaced by a spiral with toroidal mode number 8 . Thereby the heat load is deposited in many bands at any given toroidal location.

The reader is warned that Fig. 4 is misleading in an important respect. As discussed above, we employ weighted random walkers, and the typical weight of a walker is much less at larger values of $x$ than at smaller values. In contrast to the impression obtained from Fig. 4, most of the weight falls in the region between $x=$ 2.02 and $x=2.06$.

The effect of the perturbation is very visible in some reduced parameters of the distribution of hits on the boundary. The root mean square deviation (properly weighted) of the distribution of radial coordinates of hits on the lower boundary equals $3.1 \mathrm{~mm}$ in the case of no perturbation, and equals $21 \mathrm{~mm}$ in the case with perturbation. The full width at half maximum of the same distribution function equals $4 \mathrm{~mm}$ in the case of no perturbation and equals $30 \mathrm{~mm}$ in the case with perturbation. (Remember that these measures scale up by a factor of 3 when we consider ITER instead of TPX/SSAT.)

The calculation discussed above was done at a ratio $\kappa_{0} / \kappa_{\|}=10^{-8}$. For larger values of the perpendicular diffusivity the difference between cases with and without perturbation is less pronounced. However, the beneficial spreading of the heat load will be amplified in a manner not considered in the present calculation by the reduction of parallel heat conduction associated with the cooling of the plasma. We conclude that deliberate external perturbations may spread the heat load over about $0.1 \mathrm{~m}$ perpendicular to flux surfaces at the ITER divertor, even for very small values of the anomalous perpendicular diffusivity. Since this will enhance the permissible regime of operation of ITER, we recommend that this study be pursued at the level of detail required for engineering design.

A report on this work is in preparation. That report will describe in detail the optimization studies that were carried out to arrive at the particular perturbation whose effects are discussed in the previous paragraphs, and it will describe the suite of codes used for the work. We will be pleased to share these codes with other interested parties for further IrER modelling. 
Fluid Modelling of ITER Boundary Plasma. The B2 code was originally written to solve fluid equations for the edge plasma on an orthogonal mesh. This is a serious deficiency when modelling the vicinity of an inclined target plate. In previous work with David Coster of PPPL we modified the code to admit a non-orthogonal mesh, but, as we were well aware, our treatment calculates the perpendicular transport correctly only if the mesh is almost orthogonal or the parallel gradients are small. We justified that because near the target the perpendicular transport is dominated by the neutral gas, and the neutral gas dynamics may be calculated correctly by a Monte Carlo code on any mesh.

We have since implemented a fluid treatment for the neutral gas, and now it is important to discretize and solve all fluid equations correctly also on the nonorthogonal mesh. The key numerical issue is that on an orthogonal mesh our equations are of five-point molecule form, whereas on a non-orthogonal mesh they have the nine-point form. At first we are exploring the convergence of the nine-point solution algorithm when it is applied to the standard orthogonal-mesh equations. The next step is to re-write the discretization of all equations solved in the code for the more general mesh. 


\section{Figure Captions.}

Fig. 1: SSAT/TPX equilibrium \#92047, courtesy of R. Bulmer. For the purpose of our studies a relevaut ITER geometry is obtained by scaling all dimensions up by a factor 3.

Fig. 2: The flux surfaces associated with our wire model of the equilibrium. The two pairs of three circles on the outboard side indicate the rings of locations of the perturbing coils for the study presented in the text. At each toroidal location, the three magnetic dipoles have relative magnitude $(1,-2,1)$. The toroidal mode number is 8 . A toroidal phase shift between the upper and lower triplet has been optimized to get the largest effect on heat load relative to the destruction of core plasma.

Fig. 3: The Poincaré plot (on the lower inboard region of the poloidal crosssection) showing the perturbed magnetic geometry. 40 starters were followed for a maximum of 1000 toroidal transits or until the field line leaves the domain of the plot. The perturbation has destroyed the outermost $90 \mathrm{~mm}$ of flux surfaces (measured on the inboard side), and has created some islands.

Fig. 4: A scatterplot showing the location of points where the random walkers leave the domain at the lower outboard divertor (the plane $z=-1.60$ ). The $x$ coordinate represents major radius, and the $y$-coordinate represents toroidal angle (in the range $(0, \pi / 4)$ because of eightfold periodicity). 


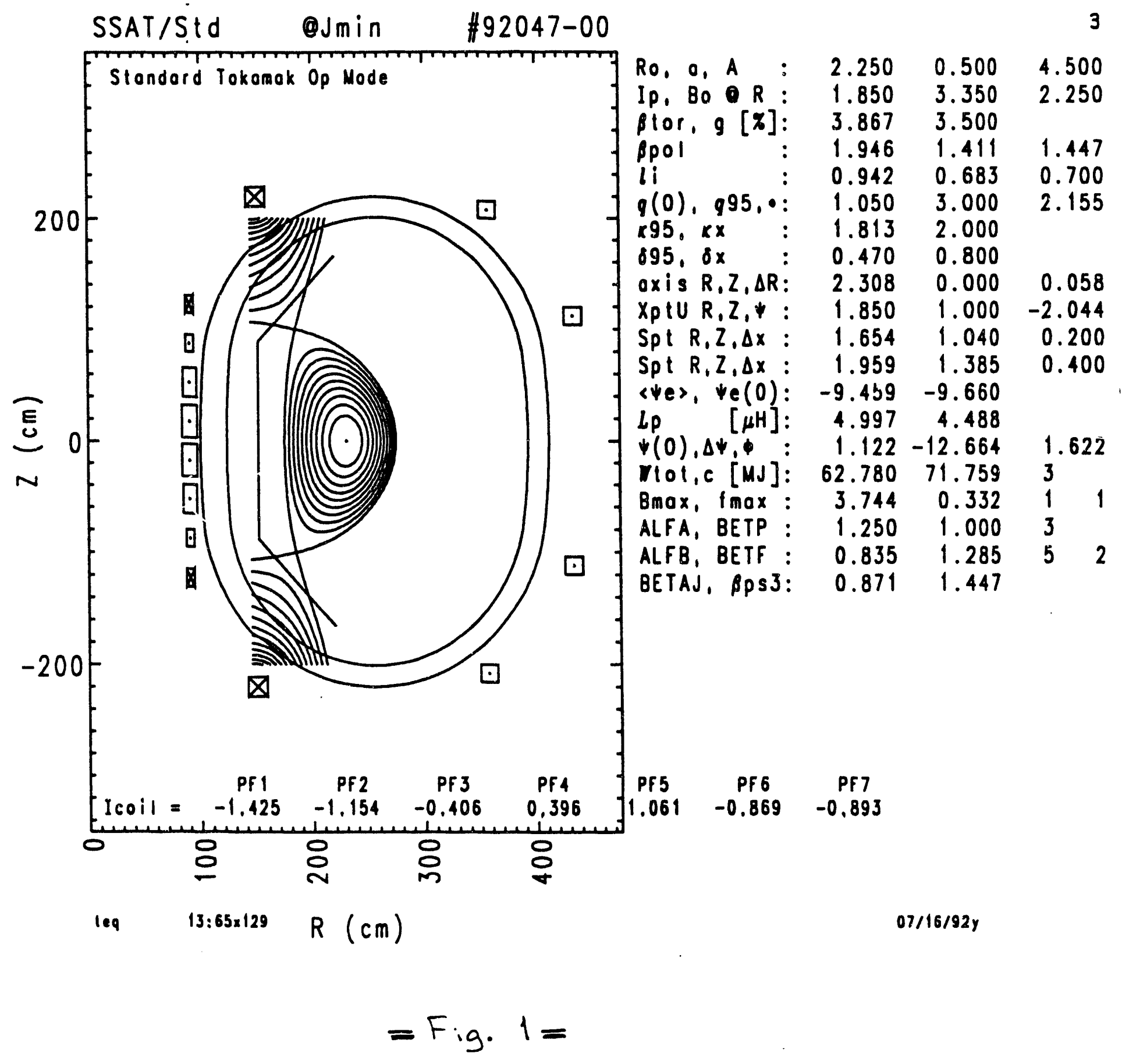




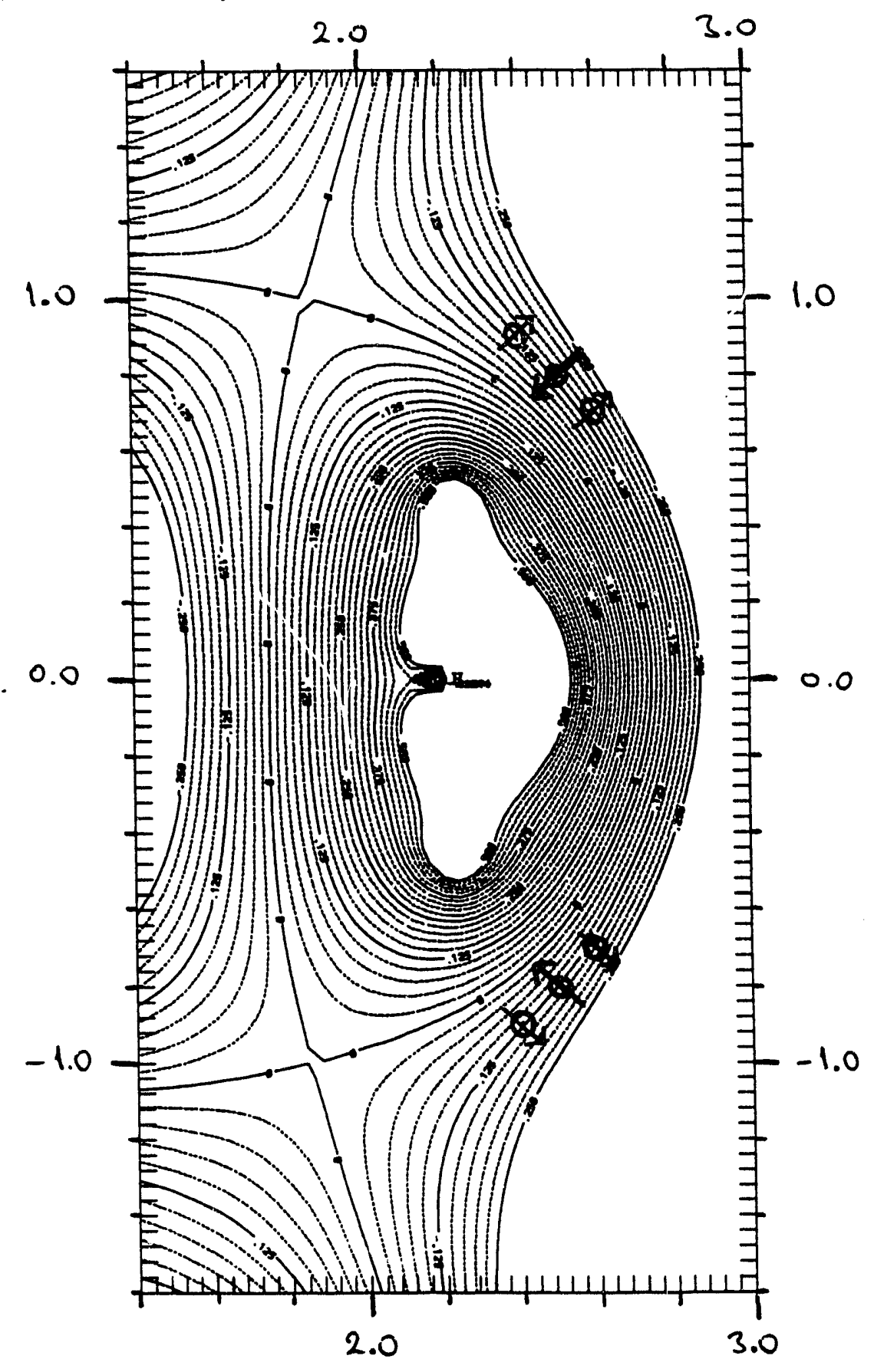

$$
=F \text { ig. } 2=
$$




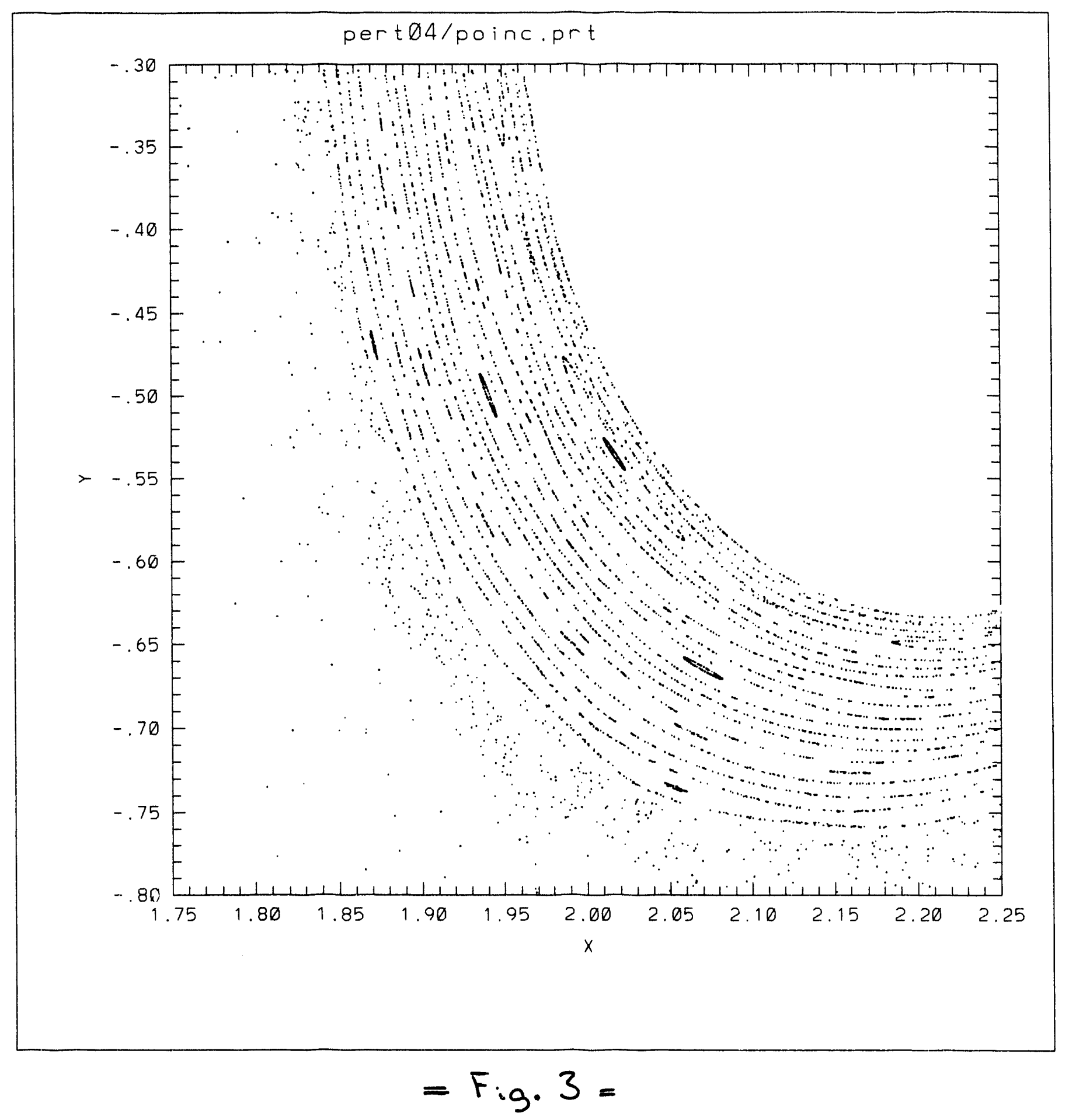




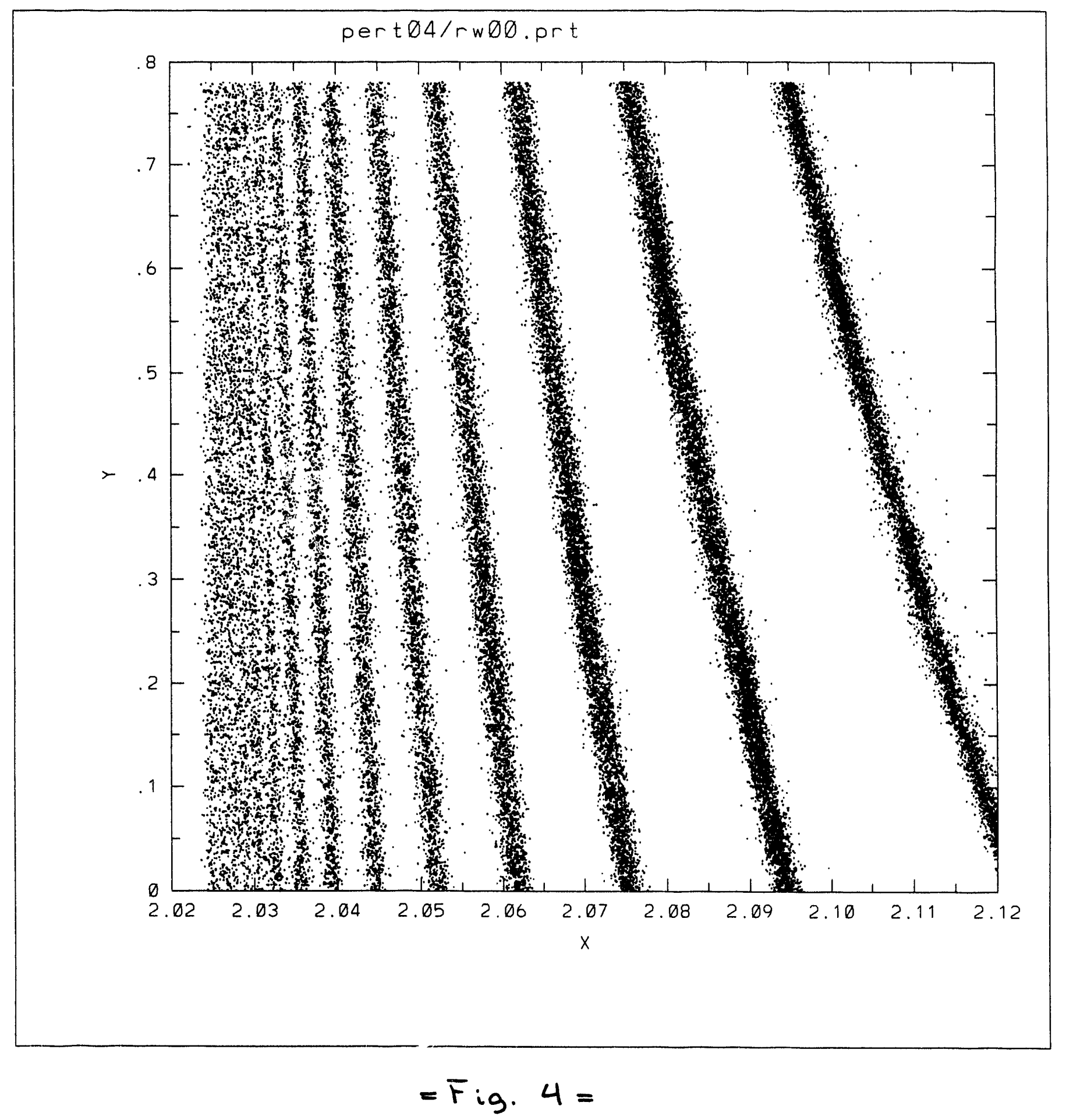



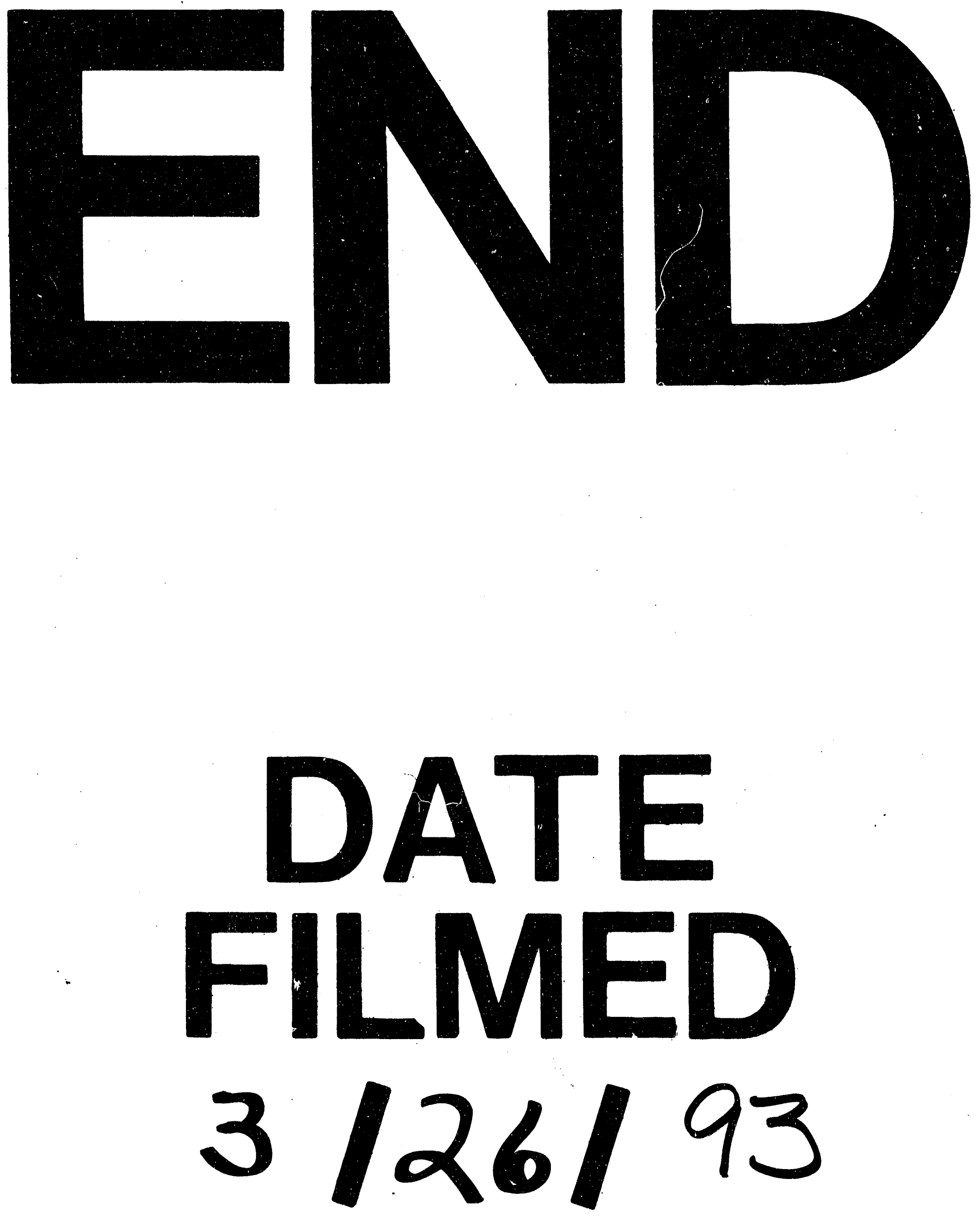
